\title{
Perfil do risco cardiovascular de mulheres adultas triadas para um programa de exercício físico
}

http://dx.doi.org/10.11606/1807-5509201800020181

\author{
Fernanda dos Santos NERI* \\ Beatriz Simões GALERA* \\ Camila Bossoni RUSSO* \\ Érick Henrique Pereira ECHES* \\ Isabella dos Santos LAQUI* \\ Crivaldo Gomes CARDOSO JÚNIOR*
}

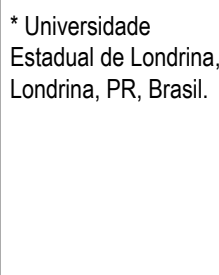

\section{Resumo}

0 objetivo do estudo foi descrever o perfil de risco cardiovascular (RCV) de mulheres adultas triadas para participação de um programa de exercício físico. Foram avaliadas 409 mulheres, com idade entre 20 e 59 anos. Seguiu-se a recomendação de triagem e estratificação do RCV proposta pelo Colégio Americano de Medicina Esportiva por meio da aplicação de um questionário face a face que identificou o Risco Alto (RA) pela presença de doença cérebro-cardiovascular ou sintomas de instabilidade cardíaca, Risco Moderado pela presença de três ou mais fatores de risco e o Risco Baixo pela presença de até dois fatores de risco para mulheres com idade inferior a 55 anos. Entretanto, para atender ao desfecho primário do estudo (prevalência do alto risco) foram agrupadas as categorias de risco baixo e moderado (RBM). A frequência das mulheres para o RA foi de $35,2 \%$, sendo: $22,9 \%$ cardiopatas, $56,9 \%$ apresentavam dispneia, $45,1 \%$ vertigem e $32,6 \%$ dor no peito. A frequência de mulheres classificadas como RBM, foi de $64,8 \%$. 0 fator de risco mais prevalente foi a obesidade, sendo $47,5 \%$ no RBM e $61,8 \%$ no RA, seguido da hipertensão para o RA $(43,1 \%)$. Houve correlação entre RA e hipertensão $(r=0,193 ; p<0,01)$; diabetes $(r=0,108$; $p<0,05)$; obesidade $(r=0,158 ; p<0,01)$ e sintomatologia climatérica $(r=0,150 ; p<0,01)$. Para o $R A$, não houve diferença estatisticamente significante na proporção de mulheres que se autodeclaram hipertensas $\left(x^{2}=0,071 ; p>0,05\right)$ e diabéticas $\left(x^{2}=0,500 ; p>0,05\right)$. Entretanto, houve diferença significante em favor de uma maior proporção de mulheres obesas e que foram classificadas na condição cardiovascular de $R A\left(x^{2}=6,367 ; p=0,01\right)$. Em conclusão, há grande prevalência de mulheres classificadas como $R A$, reforçando a importância da triagem pré-participação ao exercício.

Palavras-Chave: Atividade motora; Saúde da mulher; Cardiopatias; Fatores de risco.

\section{Introdução}

A alta mortalidade e morbidade oriunda das doenças cardiovasculares no Brasil, reconhecidas como condições crônicas e que degeneram paulatinamente o sistema cardiovascular, sobretudo quando se manifesta de forma prematura em adultos, têm importantes repercussóes na qualidade de vida e na rede social dos acometidos, além do grande impacto nos sistemas de saúde ${ }^{1}$. Parte deste sério problema de saúde pública é devido à adoção do comportamento sedentário que se configura como indicador independente de mortalidade geral e cardiovascular, resultando na morte de 1,9 milhóes de pessoas/ano no mundo 2 .
Com a criação do núcleo de apoio à saúde da família e, sobretudo com a inclusão do profissional de Educação Física na atenção primária à saúde a partir do ano de 2008, foram criadas estratégias para mobilizar as comunidades atendidas por unidades básicas de saúde (UBS) a se engajarem em programas que vislumbram a adoção de um estilo de vida fisicamente mais ativo. Para tanto, recomenda-se que seja realizada, precedentemente à prática do exercício físico moderado a vigoroso, uma triagem e estratificação do risco cardiovascular, possibilitando a adesão imediata às categorias de risco cardiovascular baixo a moderado e, para o 
alto risco cardiovascular, somente após a devida avaliação médica acompanhada de exames que possam subsidiar o controle individualizado da intensidade do esforço físico, uma vez que a exposição ao esforço físico vigoroso pode precipitar desfechos cardiovasculares indesejados ${ }^{3-5}$.

Estudos que avaliaram o risco cardiovascular à prática do exercício físico em frequentadores de parques públicos ${ }^{6}$, encontraram prevalências de $13 \%$ à $22 \%$ para o alto risco, ou seja, uma parcela considerável dos praticantes estariam inadvertidamente engajados à prática sem uma devida avaliação médica subsidiada por exames complementares. Nesse sentido, o conhecimento da prevalência e da magnitude dos principais fatores de risco para doença cardiovascular, bem como o reconhecimento da necessidade de implantação de medidas capazes de modificar tais fatores, é o primeiro passo para reduzir os efeitos deletérios sobre o sistema cardiovascular e intervir de forma efetiva nessa realidade ${ }^{7}$.

Em que pese a observação de que, convencionalmente, mulheres adultas são as principais frequentadoras de UBS, poucos estudos objetivaram avaliar o risco cardiovascular precedente a prática do exercício físico em mulheres adultas, o que perfaz uma importante lacuna científica.

Em suma, hipotetiza-se que possa existir um número expressivo de mulheres adultas, frequentadores de UBS e que buscam o engajamento à prática de programas de exercício físicos, que figuram no alto risco cardiovascular. Diante do exposto, este estudo tem por objetivo descrever o risco cardiovascular de mulheres adultas triadas para um programa de exercício físico conduzido em UBS.

\section{Método}

O estudo se caracteriza como transversal e foi desenvolvido para atender, por demanda espontânea, mulheres adultas atendidas por Unidades Básicas de Saúde (UBS) das cidades de Londrina e Cambé - PR. Este projeto foi aprovado pelo comitê de Ética e Pesquisa com seres humanos da Universidade Local (processo no 127/2013), e todas as participantes assinaram um termo de consentimento livre e esclarecido.

Inicialmente, foram realizadas afixações de cartazes, faixas, anúncios em rádios locais, distribuição de folhetos em creches, escolas públicas e privadas, comércio e igrejas das respectivas áreas de abrangência de cada uma das quatro UBS participantes, sendo duas delas na cidade de Londrina (UBSs Aquiles Stenghel e Armindo Guazzi) e as outras duas na cidade de Cambé (UBSs Ana Rosa e Novo Bandeirantes), com o intuito de cadastrar mulheres na faixa etária de 20 a 59 anos para participar de um programa de condicionamento físico somado a uma abordagem multiprofissional com vistas aos cuidados da saúde da mulher (queixa ginecológica, uso de contraceptivos, menstruação, câncer de mama ou útero, exames preventivos, autoexame das mamas, pré-natal e puerpério, amamentação e climatério).

Ao todo, compareceram 492 mulheres, sendo 314 de Londrina (Armindo Guazzi n=196; Aquiles Stenghel $\mathrm{n}=118$ ) e 178 de 178 de Cambé (Ana Rosa $\mathrm{n}=127$; Novo Bandeirantes $\mathrm{n}=51)$ ). Posteriormente foram excluídas 83 mulheres por não atenderem aos critérios de inclusão (idade entre 20 e 59 anos), de modo que a amostra final para o estudo foi composta por 409 mulheres.

A triagem pré-participação ao programa de exercício físico foi realizada pelos mesmos avaliadores, previamente treinados, em todas as UBSs e ocorreu nos períodos de março a maio de 2013. Para o risco cardiovascular relacionado à prática de exercício físico, seguiu-se a recomendação de triagem e estratificação proposta pelo American College of Sports Medicine ${ }^{8}$, em Risco Alto (RA): presença de doença cérebrocardiovascular e/ou sintomas de instabilidade cardíaca; Risco Moderado: presença de três ou mais fatores de risco; Risco Baixo: presença de dois ou menos fatores de risco para mulheres com idade inferior a 55 anos. Entretanto, para atender ao desfecho primário do estudo (identificar a prevalência de mulheres com alto risco à prática do exercício físico) foram agrupadas as categorias de risco baixo e moderado (RBM).

Para avaliação dos sintomas de instabilidade cardíaca, foram considerados: i) presença de cardiopatia - doença diagnosticada, utilização de medicamentos específicos ou históricos de cirurgias cardíacas; ii) angina - dor precordial em condição de emoção ou exercício; iii) dispneia - falta de ar desproporcionalmente aumentada diante de atividades habituais; e iv) vertigem - tontura ou síncope durante a prática de exercício. 
Para avaliação dos fatores de risco cardiovasculares, foram considerados: i) relação sexo/idade - mulher com idade superior a 55 anos; ii) histórico familiar (pai, mãe ou irmãos com histórico de cardiopatia ou morte súbita); iii) diabetes - doença diagnosticada ou utilização de medicamentos específicos; iv) dislipidemias - doença diagnosticada ou utilização de medicamentos específicos; v) hipertensão arterial sistêmica - doença diagnosticada ou utilização de medicamentos específicos; vi) tabagismo atual ou pregresso em seis meses; vii) obesidade demarcada pelo índice de massa corporal (IMC) superior a $30 \mathrm{~kg} / \mathrm{m}^{2}$; viii) pós-menopausa (ausência da menstruação retroativa em um ano ou mais) acompanhada de sintomas climatéricos. Todos os dados foram autor referidos, exceto a obesidade, que foi identificada pelo IMC, através da medida da massa corporal total $(\mathrm{kg})$ - com auxílio de uma balança mecânica - e estatura $(\mathrm{cm})$ - com auxílio de um estadiômetro acoplado à balança.

A análise da sintomatologia climatérica foi realizada por meio do inventário nomeado como índice menopausal de Kupperman. Brevemente, este índice avalia: i) sintomas vasomotores (fogachos ou sudorese noturna, pontuando em leve $=4$, moderado $=8$ ou severo $=12$; ii) parestesia, insônia e nervosismo, pontuando em leve $=2$, moderado=4 e severo=6); iii) melancolia, vertigem, fraqueza, atralgia ou mialgia, cefaleia e palpitaçáo, pontuando em leve $=1$, moderado $=2 \mathrm{e}$ severo=3). Para a classificaçáo final, foi empregada a discriminação dicotômica com valor de corte em 35 pontos?.

As fichas de triagem foram tabuladas no programa Epidata versão 3.1 por dois pesquisadores independentes e após a conferência de tabulação, os dados foram exportados para o programa Statistical Package for the Social Sciences (SPSS - versão 17.0).

Para as variáveis escalares, os dados foram testados quanto à sua normalidade pelo teste Kolmogorov-Smirnov, seguida da identificação de valores atípicos (outliers) por meio de análise gráfica (box-plot). A idade e o perfil antropométrico foram analisados em média e desvio padrão, e comparados pelo teste $t$ de Student bi-caudal para amostras independentes (grupo alto risco vs. risco baixo/moderado), considerando como diferença estatisticamente significante o $p<0,05$. Para as variáveis categóricas foram consideradas as condições de presença (código 1) ou ausência (código 2) na identificação dos fatores de risco cardiovascular. Esses fatores foram analisados quanto à sua distribuição de frequências (absolutas e relativas) nos dois grupos (grupo alto risco vs. risco baixo/moderado). Para verificar a associaçáo entre os fatores de risco cardiovascular e o alto risco à prática do exercício físico foi empregado o teste do qui-quadrado $\left(X^{2}\right)$ e para a análise do grau de correlação, foi empregado o teste de correlação de Spearman. Em todas as circunstâncias, adotouse o nível de significância inferior a 5\%.

\section{Resultados}

Ao todo, 409 mulheres participaram do estudo, sendo que a distribuiçáo dessas mulheres de acordo com a classificação do risco cardiovascular foi de 265 mulheres $(64,8 \%)$ para o RBM e 144 (35,2\%) para o RA. Dentre as classificadas no RA, os aspectos mais relevantes foram: presença de sintomas cardíacos (56,9\% para dispneia desproporcional ao esforço, $45,1 \%$ vertigem durante a prática e $32,6 \%$ dor precordial) e a presença de doença cardíaca já diagnosticada (22,9\%). Por outro lado, no RBM a maior parte das mulheres $(70,2 \%)$ apresentou a agregação da idade inferior a 55 anos com a presença de, no máximo, dois fatores de risco cardiovascular adicionais.

A caracterização e comparação das mulheres com RBM e RA, de acordo com a idade, peso, altura, índice de massa corpórea e a distribuição dos fatores de risco cardiovascular, estão apresentadas na TABELA 1.

É importante destacar que dentre os fatores de risco, os mais prevalentes em ambas as situaçóes (RBM e RA) foram a obesidade e a hereditariedade. No entanto, para o grupo RBM a hierarquização subsequente dos fatores de risco presentes foi composta de menopausa, hipertensão arterial, dislipidemia, idade $>55$ anos, tabagismo e diabetes, ao passo que no grupo de RA esta hierarquização foi composta de hipertensão arterial, menopausa, dislipidemia, idade $>55$ anos com tabagismo, e diabetes.

A FIGURA 1 representa a proporção de mulheres que manifestaram a presença do fator de risco cardiovascular em seus respectivos grupos. Como pode ser verificado, a proporçáo de mulheres com idade superior à 55 anos, dislipidêmicas, tabagistas 
e na pós-menopausa foi semelhante entre os grupos $\left(X^{2}=3,8415, \mathrm{p}>0,05\right)$. Todavia, a associação de mulheres obesas $\left(X^{2}=7,607, \mathrm{p}<0,05\right)$, hipertensas $\left(X^{2}=14,959, \mathrm{p}<0,05\right)$, diabéticas $\left(X^{2}=6,738\right.$, $\mathrm{p}<0,05)$ e com componente hereditário positivo para desfechos cardiovasculares fatais e não fatais $\left(X^{2}=91,640, \mathrm{p}<0,05\right)$ foi estatisticamente maior no RA do que no RBM.

TABELA 1 - Caracterização da amostra de acordo com a classificação de risco cardiovascular e prevalência de fatores de risco

\begin{tabular}{lcc}
\hline & Risco baixo e moderado $(\mathbf{n}=\mathbf{2 6 5})$ & Risco alto $(\mathbf{n}=\mathbf{1 4 4})$ \\
\hline Idade & $43 \pm 10$ & $42 \pm 11$ \\
Peso & $76,6 \pm 15$ & $81,0 \pm 16,6^{*}$ \\
\hline & Risco baixo e moderado $(\mathbf{n}=\mathbf{2 6 5})$ & Risco alto $(\mathbf{n}=\mathbf{1 4 4})$ \\
\hline Altura & $1,59 \pm 0,06$ & $1,58 \pm 0,07$ \\
IMC & $30,2 \pm 5,7$ & $32,1 \pm 6,2^{*}$ \\
\hline \multicolumn{3}{l}{ Prevalência de fatores de risco $-\mathbf{n}(\mathbf{\%})$} \\
Idade $>\mathbf{5 5}$ anos & $33(12,5 \%)$ & $21(14,6 \%)$ \\
DM & $21(45,7 \%)$ & $71(49,3 \%)$ \\
DLP & $14(5,3 \%)$ & $18(12,5 \%)$ \\
HAS & $53(20,0 \%)$ & $36(25,0 \%)$ \\
Tabagismo & $65(24,5 \%)$ & $62(43,1 \%)$ \\
Menopausa & $26(9,8 \%)$ & $21(14,6 \%)$ \\
Obesidade & $75(28,3 \%)$ & $47(32,6 \%)$ \\
\hline DM: Ditariedade & $126(47,5 \%)$ & $89(61,8 \%)$ \\
\hline
\end{tabular}

DM: Diabetes Mellitus; DLP: dislipidemia; HAS: hipertensão arterial sistêmica. ${ }^{*} \mathrm{p}<0,05$

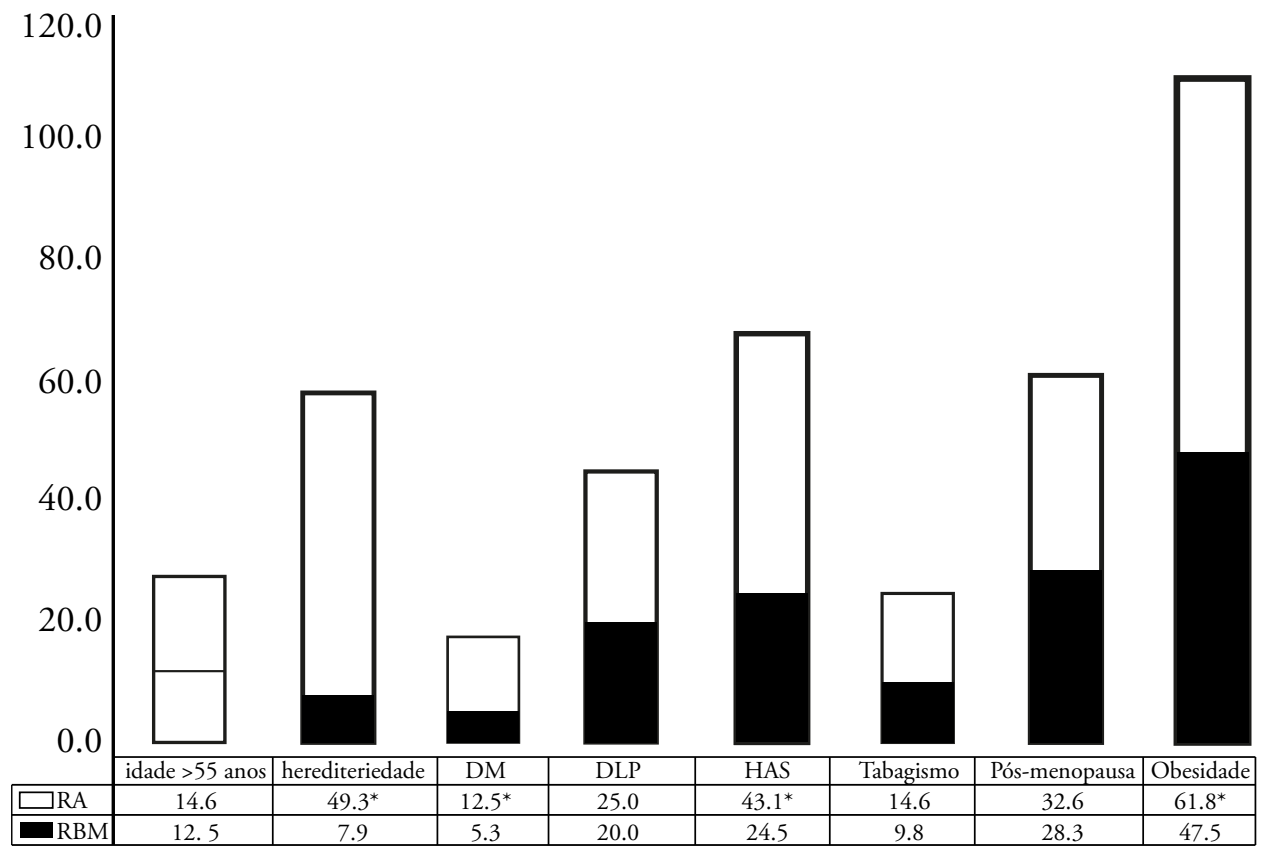

FIGURA 1 - Proporção da prevalência dos fatores de risco para o Risco Alto e Risco Baixo/Moderado. RA: Risco Alto; RBM: Risco Baixo/Moderado; DM: Diabetes Mellitus; DLP: Dislipidemia; HAS: Hipertensão Arterial Sistêmica. * $\mathrm{p}<0,05$. 
Ao analisar isoladamente o grupo com RA, foi possível verificar que não houve diferença estatisticamente significante na proporção de mulheres que se autodeclaram hipertensas $\left(X^{2}=0,071 ; p>0,05\right)$ e diabéticas $\left(X^{2}=0,500 ; p>0,05\right)$. Entretanto, houve diferença significante em favor de uma maior proporçáo de mulheres obesas e que foram classificadas na condição cardiovascular de $\mathrm{RA}\left(\mathrm{X}^{2}=6,367 ; \mathrm{p}=0,01\right)$. Em relação ao índice menopausal de Kupperman, somente sete mulheres, sendo duas delas classificadas no RA, que relataram presença de sintomas de maneira severa ( $>35$ pontos), de tal maneira que não foi possível fazer a extrapolação dos dados para a associação com o RA por se tratar de um número pouco expressivo. Por outro lado, ao analisar os dados de sintomatologia climatérica leve à moderada (até 34 pontos), foi possível verificar que a associação de mulheres com RBM e sintomas leve/moderado $(61,3 \%)$ é maior do que as mulheres com RA e sintomas leve/moderado $(38,7 \%), X^{2}=4,742, \mathrm{p}=0,02$.

Para testar a existência de relacionamento linear entre os fatores de risco cardiovascular no grupo RA foi empregada a correlação de Spearman que revelou que a idade $\left(r_{s}=0.063\right)$, a hereditariedade $\left(r_{s}=0.008\right)$, a dislipidemias $\left(r_{s}=0.075\right)$, o tabagismo $\left(r_{s}=0.082\right)$ e a menopausa $\left(r_{s}=0.045\right)$ não se relacionam com o RA ( $p>0,05)$. Porém, a obesidade $\left(r_{s}=0.158\right)$, o diabetes $\left(r_{s}=0.108\right)$, a hipertensão $\left(r_{s}=0.193\right)$ e a sintomatologia climatérica de Kupperman $\left(r_{s}=0.150\right)$ se relacionaram positivamente e significantemente com o RA $(\mathrm{p}<0,05)$.

\section{Discussão}

O presente estudo teve por objetivo descrever o risco cardiovascular de mulheres triadas para um programa de exercício físico conduzido em UBSs nas cidades de Londrina e Cambé -PR. Os principais resultados encontrados foram que: i) Há um número expressivo de mulheres $(35,2 \%)$ que figuram na classificação de alto risco cardiovascular e que deveriam ser engajadas na prática regular e supervisionada do exercício físico moderado a vigoroso somente após a liberação médica consubstanciada por avaliaçóes cardiológicas.

As Unidades Básicas de Saúde se configuram como a condição mais incipiente para a inclusão e início de tratamento de saúde vinculado ao Sistema Único de Saúde no Brasil. Como preceitua a atenção básica, a missão das UBSs é desenvolver açóes de prevenção, promoção e recuperação da saúde, de modo a intervir no processo saúde doença da população, respeitando, para tanto, os princípios da universalidade, acessibilidade, humanização, equidade e da participaçáo social ${ }^{10}$. Neste contexto, o combate ao sedentarismo e a promoção de programas de exercícios físicos se destacam como medidas necessárias para prevenir, promover e recuperar a saúde da população, sobretudo por conta dos indicativos científicos que revelam associação entre o dispêndio energético induzido pela prática da atividade física e do exercício físico com a redução da morbidade e da mortalidade por todas as causas ${ }^{11}$.

Os serviços prestados nas atençôes básicas devem assegurar níveis crescentes de complexidade e garantir formas de acesso a serviços que façam parte da diversidade requerida pelo caso nos limites dos recursos disponíveis numa dada regiāo ${ }^{12}$. Com isto, espera-se que sejam atendidas nas UBSs as mulheres com níveis de complexidade coerentes com uma condição de saúde estável e, restringindo aos níveis de maior complexidade (hospitais, por exemplo), as mulheres com condições instáveis de saúde. Entretanto, no presente estudo verificou-se que um número expressivo de mulheres apresentou alto risco cardiovascular, sendo que na maior parte dos casos o alto risco cardiovascular foi triado pela presença de sintomas cardíacos que denotam a instabilidade cardiovascular e colocam a mulher na eminência de um evento cardíaco fatal, a saber: dispneia desproporcional ao esforço $(56,9 \%)$, vertigem durante a prática do esforço físico $(45,1 \%)$ e dor precordial característica $(32,6 \%)$.

É importante destacar que a triagem e estratificação de risco cardiovascular a partir da presença de sintomas e doenças cardiovasculares torna possível identificar indivíduos com alto risco cardiovascular e, nesses casos, a mulher deveria realizar uma avaliaçáo médica antes do início da prática de exercício físico ${ }^{13}$, uma vez que na presença do alto risco cardiovascular o exercício físico pode potencializar a ocorrência de eventos cardiovasculares agudos, tais como arritmias complexas e isquemia miocárdica ${ }^{14}$. Em que pese a carência de estudos com a descrição do perfil de risco cardiovascular em frequentadoras de UBSs, um estudo similar de estratificação de 
risco, envolvendo homens e mulheres verificou prevalência de $28 \%$ para o risco alto, corroborando com os achados do presente estudo ${ }^{15}$.

Em relação à prevalência dos fatores de risco para essa amostra, o mais prevalente em ambas as situaçóes (RBM e RA), foi a obesidade com $47,5 \%$ e $61,8 \%$ respectivamente. Dados da Sociedade Brasileira de Cardiologia apontam que a prevalência de sobrepeso/obesidade no Brasil para todas as idades é de 57,9\%, variando de 50\% nas regióes Norte/ Centro-Oeste a $59,8 \%$ na região Sudeste ${ }^{16}$. Estes resultados ratificam a importância de se investir em programas de prevenção e tratamento dessa patologia, já que ela age indiretamente no aumento do risco de doenças cardiovasculares, uma vez que eleva a pressão arterial, altera desfavoravelmente o metabolismo dos lípides sanguíneos, aumenta a resistência à insulina, aumenta a sintomatologia climatérica e culmina com a maior incidência de eventos cardiovasculares fatais e não fatais ${ }^{17}$.

Ao longo da vida da mulher já é conhecido que o risco cardiovascular aumenta, principalmente na pós-menopausa fase que ocorre a diminuição dos níveis circulantes de estrogênio. Esta queda é associada ao aparecimento de doença arterial coronária, disfunção endotelial e até hipertensão ${ }^{18}$. Além dessas associaçóes, na pós-menopausa é constatado aumento na incidência de obesidade, diabetes e dislipidemias. Esses fatores, isolados ou em conjunto nas mulheres, predispóem ao desenvolvimento da doença arterial coronária e causam grande alerta para saúde pública, pois aumentam a morbimortalidade cardíaca ${ }^{19}$.

Embora a hipertensão tenha alcançado uma proporção alta para o grupo do Risco Alto, a proporção de mulheres que autodeclaram a presença da hipertensão arterial não foi superior à de mulheres que não declararam serem hipertensas no grupo RA. Segundo o Ministério da Saúde, aproximadamente 17 milhóes de brasileiros são portadores de hipertensão e sua prevalência varia de $22,3 \%$ a $43 \%$ na população adulta ${ }^{20}$. Porém, sabe-se que esta estatística é subestimada, sobretudo pelo fato da hipertensão arterial, na maior parte das vezes, ser uma patologia assintomática ${ }^{21}$ e o diagnóstico somente pode ser definido pela medida da pressão arterial ${ }^{22}$. No presente estudo, por limitações técnicas, não foi possível considerar os valores obtidos nas medições da pressão arterial. Diante disto, é possível que o impacto da hipertensão nesta amostra possa ser ainda maior, considerando que a maior causa de morte entre as mulheres brasileiras é o acidente vascular encefálico que, por sua vez, tem a hipertensão arterial como o principal fator de risco.

Em relação à prevalência de dislipidemia, o presente estudo identificou taxas menores do que se espera para mulheres brasileiras com idade entre 25 e 45 anos (40\%), porém isso pode ser explicado pelo fato do questionário ter envolvido apenas a presença das alteraçóes dos lípides sanguíneos de forma autodeclarada, o que certamente subestimou o resultado ${ }^{23}$. O diabetes por sua vez, é representado por $7,6 \%$ na população brasileira ${ }^{24}$, aliado com a hipertensão se torna responsável pela primeira causa de mortalidade e de hospitalização cardiovascular, de amputaçóes de membros inferiores e representa ainda $62,1 \%$ dos diagnósticos primários em pacientes com insuficiência renal crônica submetidos à diálise ${ }^{20}$. No presente estudo a prevalência do diabetes variou de $5,3 \%$ a $12,5 \%$ para o RBM e RA, respectivamente. Diante disto, é possível presumir que a proporção de RA no presente estudo ainda foi subestimada.

Um estudo que buscou comparar a sensibilidade de dois instrumentos PAR-Q (questionário de prontidão para atividade física) e o disponibilizado pelo ACSM verificou que o do ACSM identificou indivíduos com alto risco cardiovascular, ao passo que o PAR-Q não foi sensível o suficiente para identificar tais sujeitos e ainda sugeriu a utilização do documento do ACSM para identificação de indivíduos com doenças cardiovasculares ${ }^{25}$. Portanto, esta triagem permite verificar a necessidade de avaliação médica preliminar, a compatibilidade entre o risco cardiovascular existente e a prática realizada e a exigência de monitoramento e/ou supervisão durante a atividade, a fim de se estabelecer as orientaçóes e condutas mais apropriadas para que os benefícios sejam obtidos ao menor risco possível ${ }^{6}$.

Frente ao exposto, recomenda-se a ampliaçáo do conhecimento epidemiológico, bem como de medidas que possam incentivar novos estudos com diferentes níveis de agregação cardiovascular, reconhecendo o impacto de ações de detecção precoce, diagnóstico, tratamento e controle do evento cardiovascular, a fim de colaborar com uma orientação metodológica que permita ser adotada pelas Unidades Básicas de Saúde e, por conseguinte, apontar novos caminhos para o enfrentamento deste problema. Em conclusão, há grande prevalência de mulheres classificadas como RA, dentre àquelas que buscam a adoçấo de um estilo de vida mais ativo por meio de programas de exercício físico ofertados em UBSs nas cidades de Londrina e Cambé-PR. 


\section{Abstract}

\section{Cardiovascular risk profile of adult women screened for a physical exercise program}

The aim of this study was to describe the cardiovascular risk profile (CRP) of adult women screened for engaged in a physical exercise program. It was evaluated 409 women (aged between 20 and 59 years). The screening for the CRP by the American College of Sports Medicine recommendations was employed in a face to face form that identify the High Risk (HR) by the presence of cerebralcardiovascular disease or cardiac instability, the Moderate Risk by the presence of three or more cardiovascular risk factors and Low Risk by the presence of two risk factors for the women below 55 years old. However, to meet the primary output (prevalence of HR) the moderate and low risk was clustered (LMR). The prevalence of women in HR was $35.2 \%$ (22.9\% cardiopathic, 56.9\% presented dyspnea, dizziness and $45.1 \% 32.6 \%$ chest pain). The prevalence of women classified in LMR was $64.8 \%$. The most prevalent risk factor was obesity (47. $5 \%$ in LMR and $61.8 \%$ in HR), followed by hypertension for the HR (43.1\%). There was correlation between HR and hypertension $(r=0.193 ; p<0.01)$; diabetes $(r=0.108 ; p<0.05)$; obesity $(r=0.158 ; p<0.01)$ and menopausal symptoms $(r=0.150 ; p<0.01)$. There was no significant difference in the proportion of women who selfdeclared as hypertensive $\left(\chi^{2}=0.071 ; p>0.05\right)$ and diabetes $\left(\chi^{2}=0.500 ; p>0.05\right)$ in the HR. However, there was a significant greater proportion of obese women classified in HR $\left(\chi^{2}=6.367 ; p<0.01\right)$. In conclusion, there is high prevalence of women classified as $\mathrm{HR}$, reinforcing the importance of pre-participation screening for the exercise program.

KEYWORDS: Motor activity; Woman's health; Heart diseases; Risk fators.

\section{Conflito de interesse}

Declaramos não haver conflitos de interesse.

\section{Referências}

1. Pereira JC, Barreto SM, Passos VMA. Perfil de risco cardiovascular e autoavaliação da saúde no Brasil: estudo de base populacional. Rev Panam Salud Publica. 2009;25(6):491-8.

2. Wilmot EG, Edwardson CL, Achana FA, Davies MJ, Gorely T, Gray LJ, et al. Sedentary time in adults and the association with diabetes, cardiovascular disease and death: systematic review and meta-analysis. Diabetologia. 2012;55(11):2895-905.

3. Merghani A, Malhotra A, Sharma S. The U-shaped relationship between exercise and cardiac morbidity. Trends Cardiovasc Med. 2015;26(3):232-40. Disponível em: http://linkinghub.elsevier.com/retrieve/pii/S1050173815001711.

4. American College of Sports Medicine, American Heart Association. Exercise and acute cardiovascular events: placing the risks into perspective. Med Sci Sport Exerc. 2007;39(5):886-97.

5. Ghorayeb N, Costa RVC, Castro I, Daher DJ, Oliveira Filho JA, Oliveira MAB. Guideline in cardiology of sport and exercise of the Brazilian Society of Cardiology and the Brazilian Society of Sports Medicine. Arq Bras Cardiol. 2013;100(1.Supl.2):1-56.

6. Roque T, Pádua P, Matheus V, Bartholomeu T, Forjaz CLM. Triagem do risco cardiovascular para a prática de exercícios físicos nos freqüentadores de um parque público. Coleção Pesquisa em Eucação Física. 2007;6(1):507-14.

7. Jardim PCBV, Gondim MRP, Monego ET, Moreira HG, Vitorino PVO, Souza WKSB, et al. Hipertensão arterial e alguns fatores de risco em uma capital brasileira. Arq Bras Cardiol. 2007;88(4):452-7.

8. American College of Sports Medicine. ACSM's guidelines for exercise testing and prescription. 8th ed. Philadelphia: Lippincott Williams Wilkins; 2009.

9. Kupperman HS, Wetchler BB, Blatt MHG. Contemporary therapy of the menopausal syndrome. J Am Mecial Assoc. 1959;171(12):103-13. 
10. Teixeira CF, Molesini JA. Gestão municipal do SUS: atribuiçóes e responsabilidades do gestor do sistema e dos gerentes de unidades de saúde. Rev Baiana de Saúde Públ. 2002;26(1/2):29-40.

11. Wen CP, Wai JPM, Tsai MK, Yang YC, Cheng TYD, Lee MC, et al. Minimum amount of physical activity for reduced mortality and extended life expectancy: a prospective cohort study. Lancet. 2011;378(9798):1244-53.

12. Gonçalves AFL, Cabrera GZ, Silva MG, Andrade JA, Digiovani RAB, Storel ILA, et al. Avaliação da qualidade de vida em mulheres com fatores de risco cardiovasculares de uma unidade básica de saúde. Colloquium Vitae. 2013;5(2):92-100.

13. Meneghelo RS, Araújo CGS, Stein R, Mastrocolla LE, Albuquerque PF, Serra SM, et al. III Diretrizes da Sociedade Brasileira de Cardiologia sobre teste ergométrico. Arq Bras Cardiol. 2010;95(5 supl. 1):1-26.

14. 14. Nelson ME, Rejeski WJ, Blair SN, Duncan PW, Judge JO, King AC, et al. Physical activity and public health in older adults: recommendation from the American College of Sports Medicine and the American Heart Association. Circulation. 2007;116(9):1094-105.

15. Forjaz CLM, Tinucci T, Bartholomeu T, Fernandes TEM, Casagrande V, Massucato JG. Avaliação do risco cardiovascular e da atividade física dos frequentadores de um parque da cidade de São Paulo. Arq Bras Cardiol. 2002;79(1):35-42.

16. Hazar M, Moreira OC, Carneiro Júnior MA, Teodoro BG, Oliveira CEP. Determinação da prevalência de fatores de risco coronariano em estudantes de Educação Física de uma faculdade privada de Minas Gerais. R Bras Ci e Mov. 2010;18(2):58-66.

17. Lima WA, Glaner MF. Principais fatores de risco relacionados às doenças cardiovasculares. Rev Bras Cineantropom Desempenho Hum. 2006;8(1):96-104.

18. Kalantaridou SN, Naka KK, Papanikolaou E, Kazakos N, Kravariti M, Calis KA, et al. Impaired endothelial function in young women with premature ovarian failure: normalization with hormone therapy. J Clin Endocrinol Metab. 2004;89(8):3907-13.

19. Welin L, Adlerberth A, Caidahl K, Eriksson H, Hansson PO, Johansson S, et al. Prevalence of cardiovascular risk factors and the metabolic syndrome in middle-aged men and women in Gothenburg, Sweden. BMC Public Health. 2008;8:403.

20. Nascimento JS, Gomes B, Sardinha AHL. Fatores de risco modificáveis para as doenças cardiovasculares em mulheres com hipertensão arterial. Rev Rene. 2011;12(4):709-15.

21. Rufino DBR, Drummond RAT, Moraes WLD. Adesão ao tratamento: estudo entre portadores de hipertensão arterial cadastrados em uma Unidade Básica de Saúde. J Health Sci Inst. 2012;30(4):336-42.

22. Dasgupta K, Quinn RR, Zarnke KB, Rabi DM, Ravani P, Daskalopoulou SS, et al. The 2014 Canadian Hypertension Education Program recommendations for blood pressure measurement, diagnosis, assessment of risk, prevention, and treatment of hypertension. Can J Cardiol. 2014;30(5):485-501.

23. Nascimento Neto RM. Atlas coraçóes do brasil. São Paulo: Sociedade Brasileira de Cardiologia; 2005.

24. Thurston RC, El Khoudary SR, Sutton-Tyrrell K, Crandall CJ, Gold E, Sternfeld B, et al. Are vasomotor symptoms associated with alterations in hemostatic and inflammatory markers? Findings from the Study of Women's Health Across the Nation. Menopause. 2011;18(10):1044-51.

25. Lopes PR, Barbosa JPAS, Lima AHRA, Miranda AS, Rodrigues LBCC, Rodrigues SLC, et al. Triagem pré-participação em exercício físico em pacientes com doença arterial periférica. J Vasc Bras. 2012;11(3):194-8.

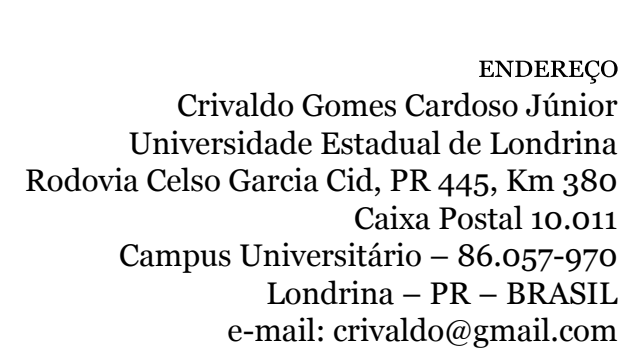

Recebido para publicação: 15/06/2015

Revisado: 26/01/2016

Aceito: 06/06/2016 